\title{
Radiofrequency ablation and percutaneous permanent iodine-125 implantation as salvage therapy for giant recurrent sclerosing epithelioid fibrosarcoma of the chest wall: A case report
}

\author{
SHAN KE, XUE-MEI DING, JUN GAO, SHAO-HONG WANG, JUN ZHANG, JIAN KONG and WEN-BING SUN \\ Department of Hepatobiliary Surgery, Beijing Chao-Yang Hospital Affiliated to Capital Medical University, \\ Beijing 100043, P.R. China
}

Received May 29, 2014; Accepted February 5, 2015

DOI: $10.3892 / \mathrm{ol} .2015 .3020$

\begin{abstract}
Sclerosing epithelioid fibrosarcoma (SEF) is a rare soft-tissue sarcoma for which there is no standardized treatment regimen available. The current treatment options for SEF are resection, radiation and chemotherapy. Surgical resection remains the mainstay of therapy for SEF. However, SEF is an aggressive tumor that is prone to repeated local recurrence if not widely excised. Radiation and chemotherapy are less commonly used due to the insensitivity of SEF to these therapies. The treatment of recurrent SEF is even more challenging. The present study describes a patient who presented with a giant recurrent SEF arising from the chest wall that was accompanied by emergent bleeding. The patient was a 70-year-old male who had multiple comorbid diseases, including hypertension and chronic cardiac dysfunction. A computed tomography (CT) scan indicated the involvement of the sternum and anterior mediastinum. However, the patient refused any further surgery. Subsequent to careful discussion and consideration, radiofrequency (RF) ablation and percutaneous iodine-125 implantation was administered. The emergent bleeding was successfully stopped and the tumor was eliminated using RF ablation. Percutaneous iodine-125 implantation under CT guidance established effective control on the growth of the tumor involving the mediastinum. Despite this, the tumor recurred 6 months after treatment. The patient refused any further treatment and was discharged. In conclusion, RF ablation and percutaneous permanent iodine-125 implantation is a feasible and safe salvage therapy for patients with recurrent SEF of the chest wall.
\end{abstract}

Correspondence to: Dr Wen-Bing Sun, Department of Hepatobiliary Surgery, Beijing Chao-Yang Hospital Affiliated to Capital Medical University, 5 Jingyuan Road, Beijing 100043, P.R. China

E-mail: cyhswb@qq.com

Key words: sclerosing, epithelioid, fibrosarcoma, recurrent, treatment

\section{Introduction}

Sclerosing epithelioid fibrosarcoma (SEF) is a rare tumor affecting the deep soft tissues that was originally described by Meis-Kindblom et al in 1995 (1). The most commonly affected sites are the lower extremities, limb girdles and trunk, followed by the upper extremities, the head and neck, and the abdominal inguinal region (2,3). The tumor may also arise in the bones, neural system, cecum, ovaries and kidneys (4-8). SEF is an unusual variant of fibrosarcoma that is formed from epithelioid cells arranged in strands and nests in a background of a highly sclerotic collagenous stroma. Although SEFs exhibit an indistinctive appearance and low mitotic activity, they are capable of distant metastasis and local recurrence. The current treatment options for SEF are resection, radiation and chemotherapy (3). Radiofrequency (RF) ablation and percutaneous permanent iodine-125 implantation have been used to treat numerous types of solid tumor, including hepatocellular carcinoma and lung cancers (9-14), however, at present, the efficacy of such modalities for the treatment of SEF remains unclear.

The present study describes a rare case of giant recurrent SEF arising from the chest wall that was accompanied by acute bleeding, and in addition, discusses the current therapeutic approaches, including RF ablation and percutaneous permanent iodine-125 implantation. Written informed consent was obtained from the patient.

\section{Case report}

A 70-year-old male presented to Beijing Chao-Yang Hospital Affiliated to Capital Medical University (Beijing, China)for RF ablation of a giant recurrent SEF of the chest wall, which was accompanied by acute bleeding. The patient had previously undergone several courses of radiotherapy, in addition to one surgical wedge resection. A diagnosis of SEF had been previously confirmed following pathological examination of surgical samples; histologically, the lesions were characterized by the proliferation of uniform, small to moderately-sized, slightly angulated, round to ovoid epithelioid cells with sparse, clear cytoplasm arranged in distinct cords and strands, embedded in dense collagenous stroma. The tumor had recurred at the original site 7 months prior to presentation, 


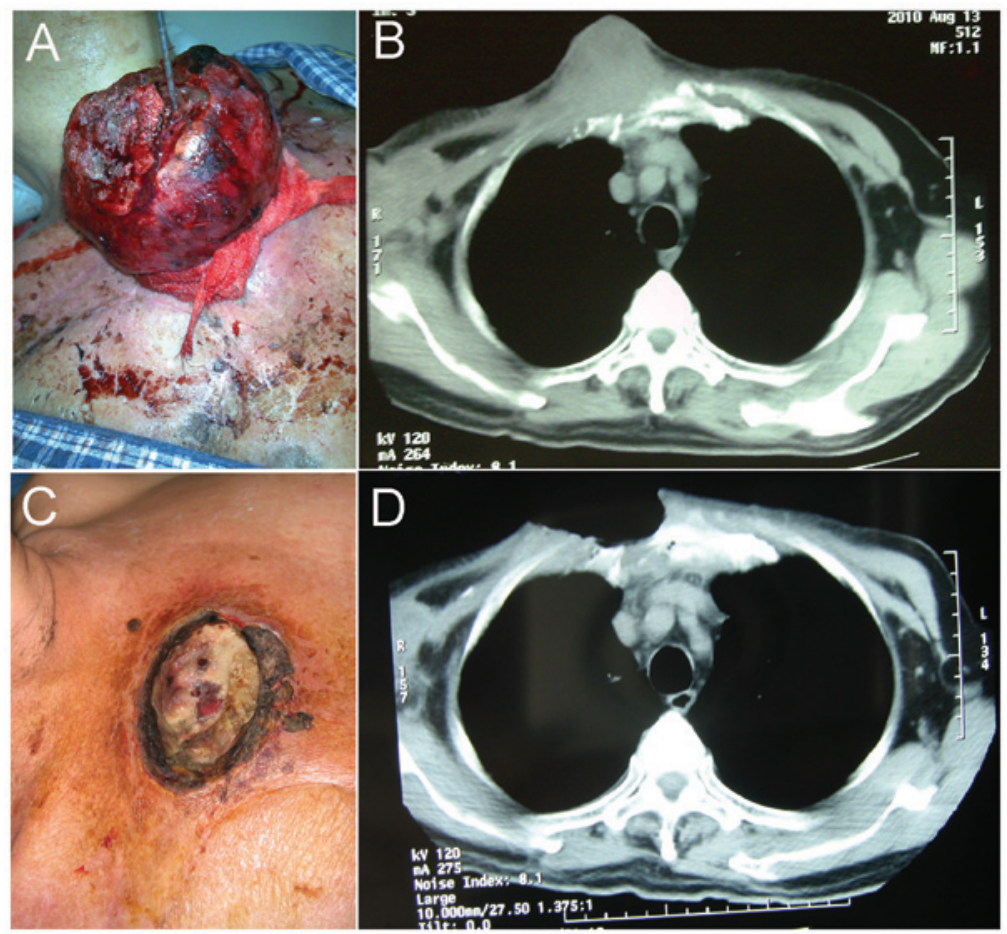

Figure 1. Emergency percutaneous radiofrequency (RF) ablation of a giant recurrent sclerosing epithelioid fibrosarcoma of the chest wall. (A) percutaneous RF ablation of the tumor also stopped the accompanying bleeding. (B) Chest computed tomography (CT) prior to RF ablation. (C) The outer region of the tumor was completely eliminated following RF ablation. (D) Chest CT subsequent to RF ablation.
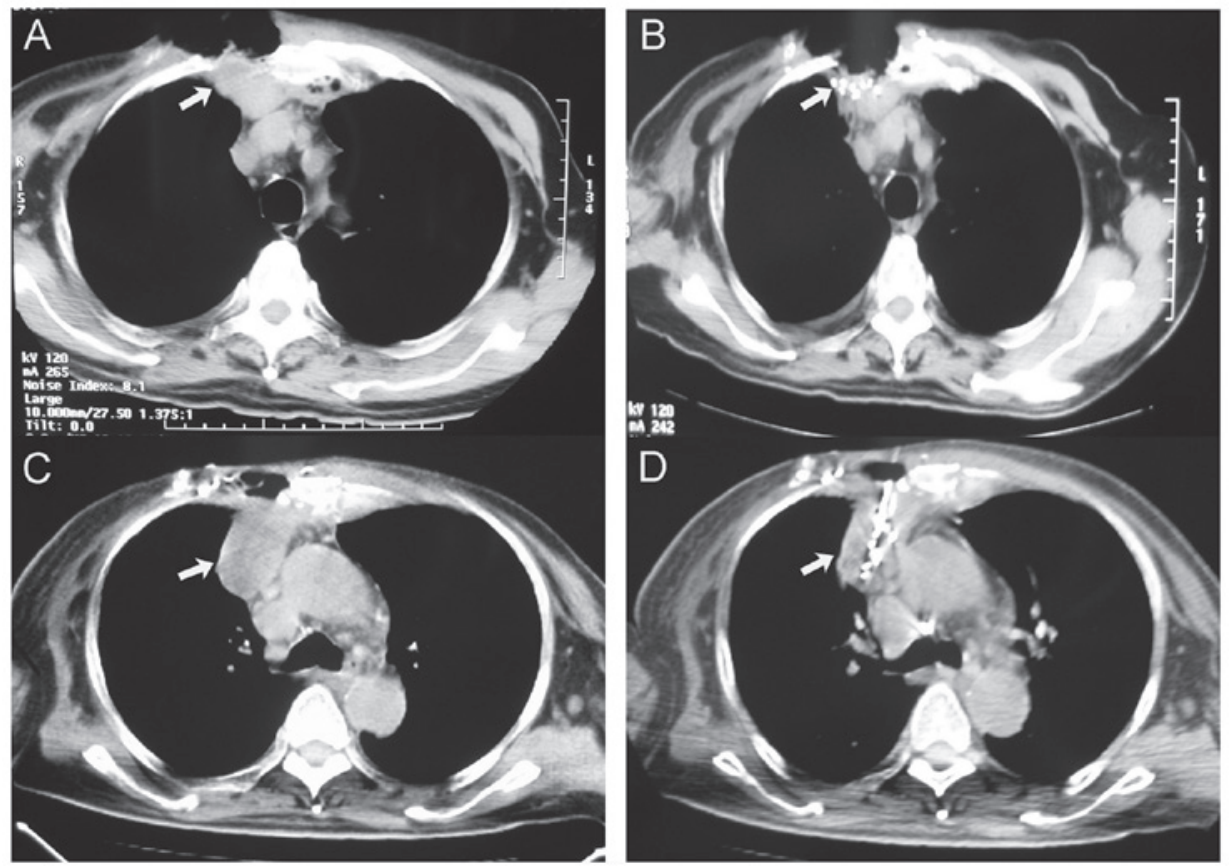

Figure 2. First and fifth chest computed tomography (CT) scans of the percutaneous iodine-125 implantation. (A) Chest CT prior to the first percutaneous iodine-125 implantation. (B) Chest CT subsequent to the first percutaneous iodine-125 implantation. (C) Chest CT scan prior to the fifth percutaneous iodine-125 implantation. (D) Chest CT scan subsequent to the fifth percutaneous iodine-125 implantation. White arrows indicate the residual tumor.

and had been gradually increasing in size. According to the patient, the tumor had increased in size by two-fold during the previous 2 months. The medical history was notable for hypertension, chronic cardiac dysfunction and benign prostatic hypotrophy. Medications included $75 \mathrm{mg} /$ day aspirin, which was discontinued 1 day prior to admission, $20 \mathrm{mg} /$ day fluvastatin, $80 \mathrm{mg} /$ day valsartan and hydrochlorothiazide, $10 \mathrm{mg}$ morphine sulfate sustained-release tablets every $12 \mathrm{~h}$, and $0.4 \mathrm{mg} /$ day tamsulosin.

At the time of admission, the patient presented with pallor and a pulse rate of $98 \mathrm{bpm}$. The patient exhibited a fungating and well-circumscribed mass on the right upper region of 
the anterior chest wall, which measured $\sim 21 \times 14 \times 5 \mathrm{~cm}$ in size (Fig. 1A and B). Bleeding was observed from the ulcerative surface of the tumor. A tamponade dressing was therefore applied in order to temporarily stop the bleeding. However, the lesion continued to hemorrhage. The patient was rehydrated to maintain effective circulation, and urgently transfused with $600 \mathrm{ml}$ of fresh frozen plasma to improve coagulation. Chest CT indicated involvement of the sternum and anterior mediastinum. Laboratory findings revealed a hematocrit level of 36\% (normal range, 40-50\%), a hemoglobin level of $10 \mathrm{~g} / \mathrm{dl}$ (normal range, 12-16 g/dl), a platelet count of 109,000 (normal range, 100,000-300,000), a partial thromboplastin time of $33.4 \mathrm{sec}$ (normal range, 35.0-45.0 sec) and a prothrombin time of $11.8 \mathrm{sec}$ (normal range, 13.0-17.0 sec). Platelet function investigations were not performed. The patient remained stable during positioning and initial CT scanning.

An emergency percutaneous RF ablation, guided by a Synergy Plus CT scanner (GE Yokogawa Medical Systems Ltd., Tokyo, Japan), was performed in a CT suite. Local anesthesia was selected, as the patient was not suitable for general anesthesia. The patient was placed in a supine position on the CT table, and the RF procedures were performed using a 15-gauge multitined electrode (Starburst XL; RITA Medical Systems, Inc., Manchester, GA, USA). The RF generator (RITA 1500, RITA Medical Systems, Inc.) was used according to the manufacturer's instructions. Multiple-spot RF ablation was first performed in order to achieve hemostasis and reduce and sclerify the lesion. The ablated tumor was then resected in a block-by-block, superficial-to-deep manner, and the outer region of the tumor was completely eliminated (Fig. 1C and D). Hemostasis was achieved by the cessation of bleeding, a decrease of the pulse rate and an increase in the hemoglobin level. In order to further control the growth of the residual tumor that had involved the mediastinum, percutaneous iodine-125 implantation under CT guidance was subsequently performed, every 30-40 days (Fig. 2). In total, five percutaneous iodine-125 implantations were performed in order to retard tumor growth. However, the tumor recurred again 6 months after treatment. The patient refused any further treatment and was discharged.

\section{Discussion}

At present, the optimal treatment strategy for SEF remains controversial. Surgical resection is the mainstay of therapy for SEF. However, SEF is an aggressive tumor that is prone to repeated local recurrence if not widely excised. It has been reported that $>50 \%$ of SEF patients experience persistent disease or local recurrence. Furthermore, the metastatic rate of SEF is between 43 and $86 \%$. In total, approximately one-third of SEF patients are alive with the disease, and the mortality rate is between 25 and $57 \%$ (2). Therefore, the first and most important step in the course of surgery is the wide excision of the tumor in order to guarantee the clearance of all microscopic tumors. The recommended margin of resection for smaller tumors is between 3 and $5 \mathrm{~cm}$, whereas for larger tumors, this should be extended to between 7 and $8 \mathrm{~cm}$. In addition, grafting is often necessary (15). Pre- or post-operative radiation therapy is considered to be an important treatment for SEF. However, not all SEFs are sensitive to irradiation. It has been previously suggested that radiation therapy should only be administered in cases where surgery is impossible, and/or where there may be the potential to slow the progress of the disease (15). Chemotherapy is less commonly administered due to the general insensitivity of SEF to this treatment.

Treatment for recurrent SEF is even more challenging. In the present study, the recurrent tumor arose from a deep site in the chest wall of a male patient. Although the tumor was well-circumscribed from the outside, distinct clinicopathological characteristics were also observed. The prominent characteristic was that the patient suffered from an emergent bleeding of the tumor that was difficult to control. The second characteristic was that the patient was 70 years old and presented with multiple comorbid diseases, including hypertension and chronic cardiac dysfunction. Thirdly, CT scans indicated that the sternum and anterior mediastinum had been involved by the tumors. Finally, the patient resolutely refused further surgery. Therefore, secondary surgery was not taken into account in the present case. Following careful discussion and consideration, it was decided that RF ablation and percutaneous iodine-125 implantation would be used to treat the patient.

RF ablation is the use of RF energy to thermally destroy living tissue. The process has gained interest as a minimally invasive strategy for the management of focal malignant diseases. Due to the fact that a number of these tumors are not responsive to curative surgical resection, RF ablation represents a novel addition to the range of available treatments. The advantages of RF ablation include real-time imaging guidance, the ability to remove tumors in patients who are unsuitable for surgical resection, a reduced risk of morbidity compared with surgical intervention, and the potential for being performed repeatedly (16). In addition, RF ablation has been used as an emergency procedure to control bleeding from ruptured tumor tissues (17-19). In the present study, not only was the tumor successfully destroyed, but the hemorrhaging was also effectively controlled following the use of RF ablation. In order to further control the growth of the tumor that had involved the mediastinum, percutaneous iodine-125 implantation was used under CT guidance. The implantation of radioactive material into tumors was established in 1901 by Pierre Curie, who used newly-discovered radium $(20,21)$. Since 1965 , iodine-125 seeds have been used. The long half-life and low energy of iodine-125 seeds have several radiobiological advantages. Firstly, the radiation hazard for members of the patient's family and involved personnel is reduced to insignificant levels. Secondly, the seeds are viable for long periods of time, and can therefore be ordered at fixed intervals and stored until use. Finally, the seeds can be applied as a permanent implant into the tumor (22). Compared with external radiation therapy, iodine-125 implantation can treat deep-seated tumors with a much higher total dose, which can be delivered more precisely and at a low dose rate.

Due to the rarity and complex spectrum of the tumors, guidelines for the treatment of recurrent SEFs have been difficult to establish. A multidisciplinary approach for the management of these patients is therefore advocated. The modality of treatment should be individually tailored based upon the analysis of the patient's situation, in addition to the tumor size, growth rate and location. Regular and careful follow-up examinations are essential and will lead, in the 
majority of patients, to a more suitable modality of treatment, which should prolong life and improve overall health. In the present case, after RF ablation and percutaneous iodine-125 implantation treatment, the patient survived for one year prior to being lost to follow-up. Despite tumor recurrence six months after therapy, treatment was considered to be satisfactory. In conclusion, the high local tumor control rates, minimal invasion and low morbidity suggest that RF ablation and percutaneous permanent iodine-125 implantation is a feasible and safe salvage therapy for patients with recurrent SEF of the chest wall.

\section{Acknowledgements}

This study was supported by grants from the Dr Jieping Wu Medical Foundation (no. 320675012712) and the Program for Medical Key Discipline of Shijingshan District (no. 20130001).

\section{References}

1. Meis-Kindblom JM, Kindblom LG and Enzinger FM: Sclerosing epithelioid fibrosarcoma. A variant of fibrosarcoma simulating carcinoma. Am J Surg Pathol 19: 979-993, 1995.

2. Antonescu CR, Rosenblum MK, Pereira P, Nascimento AG and Woodruff JM: Sclerosing epithelioid fibrosarcoma: a study of 16 cases and confirmation of a clinicopathologically distinct tumor. Am J Surg Pathol 25: 699-709, 2001.

3. Ossendorf C, Studer GM, Bode B and Fuchs B: Sclerosing epithelioid fibrosarcoma: case presentation and a systematic review. Clin Orthop Relat Res 466: 1485-1491, 2008.

4. Frattini JC, Sosa JA, Carmack S and Robert ME: Sclerosing epithelioid fibrosarcoma of the cecum: a radiation-associated tumor in a previously unreported site. Arch Pathol Lab Med 131: 1825-1828, 2007.

5. Grunewald TG, von Luettichau I, Weirich G, et al: Sclerosing epithelioid fibrosarcoma of the bone: a case report of high resistance to chemotherapy and a survey of the literature. Sarcoma 2010: 431627, 2010

6. Hanson IM, Pearson JM, Eyden BP, Slawik S and Harris M: Evidence of nerve sheath differentiation and high grade morphology in sclerosing epithelioid fibrosarcoma. J Clin Pathol 54: 721-723, 2001.

7. Watanabe K and Suzuki T: Epithelioid fibrosarcoma of the ovary. Virchows Arch 445: 410-413, 2004.

8. Argani P, Perlman EJ, Breslow NE, et al: Clear cell sarcoma of the kidney: a review of 351 cases from the National Wilms Tumor Study Group Pathology Center. Am J Surg Pathol 24: 4-18, 2000.
9. Lin ZY, Chen J and Deng XF: Treatment of hepatocellular carcinoma adjacent to large blood vessels using 1.5T MRI-guided percutaneous radiofrequency ablation combined with iodine-125 radioactive seed implantation. Eur J Radiol 81: 3079-3083, 2012.

10. Chen K, Chen G, Wang H, et al: Increased survival in hepatocellular carcinoma with iodine-125 implantation plus radiofrequency ablation: a prospective randomized controlled trial. J Hepatol 61: 1304-1311, 2014.

11. Hiraki T, Gobara H, Iguchi T, Fujiwara H, Matsui Y and Kanazawa S: Radiofrequency ablation for early-stage nonsmall cell lung cancer. Biomed Res Int 2014: 152087, 2014.

12. Niu L, Zhou L, Xu K and Mu F: Combination of cryosurgery and Iodine-125 seeds brachytherapy for lung cancer. J Thorac Dis 4: 504-507, 2012.

13. Yang H, Liu YH, Xu L and Liu LH: Efficacy of permanent iodine-125 seed implants and gemcitabine chemotherapy in patients with platinum-resistant recurrent ovarian carcinoma. Asian Pac J Cancer Prev 15: 9009-9013, 2014.

14. Jiang YL, Meng N, Wang JJ, Ran WQ, Yuan HS, Qu A and Yang RJ: Percutaneous computed tomography/ultrasonography-guided permanent iodine-125 implantation as salvage therapy for recurrent squamous cell cancers of head and neck. Cancer Biol Ther 9: 959-966, 2010.

15. Staffords ES and Ward GE: Treatment of fibrosarcoma. Ann Surg 137: 639-644, 1953.

16. Lau WY and Lai EC: The current role of radiofrequency ablation in the management of hepatocellular carcinoma: a systematic review. Ann Surg 249: 20-25, 2009.

17. Fuchizaki U, Miyamori H, Kitagawa S and Kaneko S: Radiofrequency ablation for life-threatening ruptured hepatocellular carcinoma. J Hepatol 40: 354-355, 2004.

18. Sun WB, Ding XM, Ke S, Gao J and Zhang YF: Repeated radiofrequency ablation as both salvage solution and curative treatment for spontaneous rupture of giant medial lobe hepatocellular carcinoma. Chin Med J (Engl) 122: 2067-2070, 2009.

19. Manikam J, Mahadeva S, Goh KL and Abdullah BJ: Percutaneous, non-operative radio frequency ablation for haemostasis of ruptured hepatocellular carcinoma. Hepatogastroenterology 56: 227-230, 2009.

20. Grammaticos PC: Pioneers of nuclear medicine, Madame Curie. Hell J Nucl Med 7: 30-31, 2004.

21. Schwarz SB, Thon N, Nikolajek K, Niyazi M, Tonn JC, Belka C and Kreth FW: Iodine-125 brachytherapy for brain tumours - a review. Radiat Oncol 7: 30, 2012.

22. Holm HH, Strøyer I, Hansen H and Stadil F: Ultrasonically guided percutaneous interstitial implantation of iodine 125 seeds in cancer therapy. Br J Radiol 54: 665-670, 1981. 